This paper has been accepted for publication in IEEE Symposium on Computers and Communications, June 30 - July 3, 2019 - Barcelona, Spain @ 2019 IEEE. Personal use of this material is permitted. Permission from IEEE must be obtained for all other uses, in any current or future media, including reprinting/republishing this material for advertising or promotional purposes, creating new collective works, for resale or redistribution to servers or lists, or reuse of any copyrighted component of this work in other works.

\title{
On the use of prioritization and network slicing features for mission critical and commercial traffic multiplexing in 5G Radio Access Networks
}

\author{
Shaoxuan Wang and Ramon Ferrús \\ Universitat Politècnica de Catalunya (UPC), Barcelona, Spain
}

[shaoxuan.wang, ramon.ferrus]@upc.edu

\begin{abstract}
The Public Protection and Disaster Relief (PPDR) sector is undergoing an important transition with the deployment of Mission Critical (MC) mobile broadband technology based on 3GPP standards, with multiple initiatives on-going worldwide for providing PPDR agencies with broadband communications capabilities. One common approach being adopted is the delivery of MC services together with commercial traffic over public mobile networks and the use of prioritization mechanisms to protect the MC connections in congestion situations. However, this approach leaves commercial traffic unprotected in front of a noncontrolled surge of MC traffic in specific cells since all resources would be allocated to serve this traffic. In this context, this paper proposes a solution to properly multiplex MC and commercial services with congestion protection for both types of services. The solution is based on the exploitation of the network slicing features brought into the new 5G standards. In particular, the paper describes how different slices can be parameterized in a 5G Radio Access Network (RAN) so that radio load guarantees can be established for each type of service. The proposed solution is evaluated in an illustrative scenario by means of simulations. Obtained results show the improvements in traffic isolation achievable by the slicing configuration when compared to the solution that only relies on prioritization mechanisms.
\end{abstract}

Keywords-Mission Critical Services, Prioritization, Network slicing, Public Protection, and Disaster Relief Communications

\section{INTRODUCTION}

$5 \mathrm{G}$ systems are expected to be able to simultaneously support a wide range of application scenarios and business models, resulting from the anticipated pervasive adoption of $5 \mathrm{G}$ technologies in different vertical markets[1]. One of these markets is in the Public Protection and Disaster Relief (PPDR) sector. Indeed, the requirements of PPDR operational forces are being taken into account by 3 GPP in the specification of LTE and $5 \mathrm{G}$ standards. This will ensure that the benefits of $5 \mathrm{G}-$ the modular infrastructure, use of network slicing and integration of internet of things (IoT) applications - will also support the work of public-safety agencies in the future[2]. So far, 3GPP has specified several enhancements at radio and network levels (e.g. proximity services, group communications enablers, isolated operation of a radio access network node) as well as it has produced application-level specifications of Mission Critical Services (MCS) such as Mission Critical PushTo-Talk (MCPTT), MC Video and MC Data services[3][4][5].
In this context, there are multiple initiatives currently ongoing worldwide for providing PPDR agencies with broadband communications capabilities through public or private $4 \mathrm{G} / \mathrm{LTE}$ networks. Some countries are considering whether a dedicated, state-owned PPDR mobile network should be established, citing security and government control as the main motivation for this approach. Other countries are looking at models that use existing commercial mobile networks as a basis for a PPDR service. This normally involves strengthening the robustness and security of these networks and implementing new functionality required by emergency services. Moreover, when the same network is going to be used for both commercial services and MC services, it becomes critical the adoption of measures able to guarantee the proper prioritization between both types of services and the distribution of the shared network resources.

The current prevailing approach to deal with the multiplexing of MC and commercial services over the same network is based on the exploitation of the 3GPP QoS, prioritization, and pre-emption (QPP) capabilities. At the network level, these QPP capabilities include the 5G QoS Identifier (5QI) and Allocation and Retention Priority (ARP) parameters that characterize the behavior of the data delivery services provided by the network. In this regard, the assignment of higher priority 5QI/ARP to MC services could be appropriate for situations where the aggregated MC traffic load is below the network capacity. However, in a localized emergency scenario where both $\mathrm{MC}$ and commercial traffic can surge and turn into network congestion, prioritization only based on the use of the 5QI/ARP parameters does not suffice to provide some protection to the commercial traffic so that additional mechanisms like network slicing capabilities brought into $5 \mathrm{G}$ systems become necessary. Therefore, this paper proposes a solution to handle the multiplexing of $\mathrm{MC}$ and Commercial (CO) services based on the exploitation of network slicing features.

The rest of the paper is organized as follows. Section II provides an overview of related work on exploiting prioritization mechanisms for MC services and on the use of network slicing for traffic isolation. Section III presents the proposed solution to multiplex $\mathrm{MC}$ and $\mathrm{CO}$ traffic based on network slicing, detailing both how RAN slices can be parameterized (e.g. radio load guarantees given to each type of 
services) as well as the operation of the underlying Radio Resource Management (RRM) functions supporting the radio resource distribution (e.g. admission control and scheduling). Section IV describes the evaluation scenario with the different configurations being evaluated and Section $\mathrm{V}$ provides the simulation results and discussion. Finally, Section VI draws the conclusions.

\section{RELATED WORK}

The utilization of traffic prioritization mechanisms to cope with emergency or MC services in cellular networks has been studied in multiple works. To cite a few, work in [6][7] consider resource allocation optimization problem underling their own model or architecture in 4G-LTE for public safety and commercial users running elastic or inelastic traffic by given priority over the commercial group. Different from work in [6] and [7] focus on network model and architecture, authors in [8] developed a new priority scheduling algorithm named Courteous Priority Access (CPA), which does not prevent the exhaustion of radio resources, it rather serves to delay congestion. CPA scheme is mainly sharing commercial radio for MC traffic access and scheduling in LTE heterogeneous networks. In[9], the authors propose an efficient prioritized Medium Access Control (MAC) protocol to support $\mathrm{MC}$ services for IoT applications under wireless sensor network. The proposed protocol handles the simultaneous transmissions of critical data to reduce the collision probability between the contending nodes, which in turn decreases the transmission latency.

On the other hand, less attention has been given to the utilization of network slicing features for MC traffic handling. For instance, Authors in [10]developed a new network communication architecture for RAN slicing for commercial and $\mathrm{MC}$ services, without considering prioritization mechanisms. Work in [11] tries to use licensed shared access (LSA), QoS prioritization mechanisms. and static network slicing techniques to control $\mathrm{MC}$ traffic under $5 \mathrm{G}$ network. Although the authors in [12] presented a scenario with RAN slices for commercial and MC services and proposed different options for realizing $\mathrm{MC}$ traffic differentiation (i.e. radio scheduling) and protection (i.e. admission control) by evaluating different parameters in 5G RAN slice. However, this work only analyzed the impact of commercial services overload.

In this paper, a solution that combines both slicing techniques and priority mechanisms to allocate resources to $\mathrm{MC}$ and $\mathrm{CO}$ services is considered. The performance of the solution in terms of isolation under different congestion situations for both types of traffic is evaluated and compared to the results achieved with a solution only exploiting prioritization.

\section{RAN SLICING AND RRM CONFIGURATION FOR MC AND CO TRAFFIC MULTIPLEXING}

The proposed solution to multiplex $\mathrm{MC}$ and commercial traffic is based on the configuration framework for Radio Access Network (RAN) slicing initially introduced in[13] and further developed in[12] In particular, from a functional perspective,[12] defines a set of configuration descriptors for the radio protocol layers L3, L2, and L1 of a NG-RAN cell that can be used to specify the operation of a RAN slice. This configuration framework, together with the specific realization of the descriptors consider in our work, is illustrated in Fig.1. As shown in the figure, L3 comprises the Radio Resource Control (RRC) protocol and RRM functions, such as the Radio Admission Control (RAC) for the activation and maintenance of Radio Bearers (RB), which are the data transfer services delivered by the radio protocol stack. For each UE, one or more user plane RBs, denoted as Data RBs (DRBs), can be established per Protocol Data Unit (PDU) session, which defines the connectivity service provided by the 5GC[14]. A PDU session is associated with a particular RAN slice so that the behavior of all of the DRBs established within the PDU session will be abided by the configuration of the RAN slice. When multiple RAN slices are realized over shared radio resources, the RAC has to assure that each RAN slice gets the expected amount of resources and, in case, handle any resource conflicts that might appear across slices. This idea is illustrated in Fig. 1 with two different sets of QoS flows/DRBs associated with two different RAN slices (RAN Slice $\mathrm{ID}=\mathrm{x}$ and $\mathrm{y}$ ) and subject to the RAC decisions. Hence, whenever a Guaranteed Bit Rate (GBR) DRB associated with a given RAN slice is established in a cell, the RAC process is executed to check the availability of resources in the cell and in the particular RAN slice to provide the requested bit rate guarantees. Accordingly, this paper considers as the main means of RAN slices' control related to L3 a parameter that specifies the maximum percentage of Physical Resource Blocks (PRBs) that can be considered in the RAC for the admission of GBR DRBs within the slice. This parameter is denoted as $\theta_{A C}^{\max }(s)$ for the slice $s$.

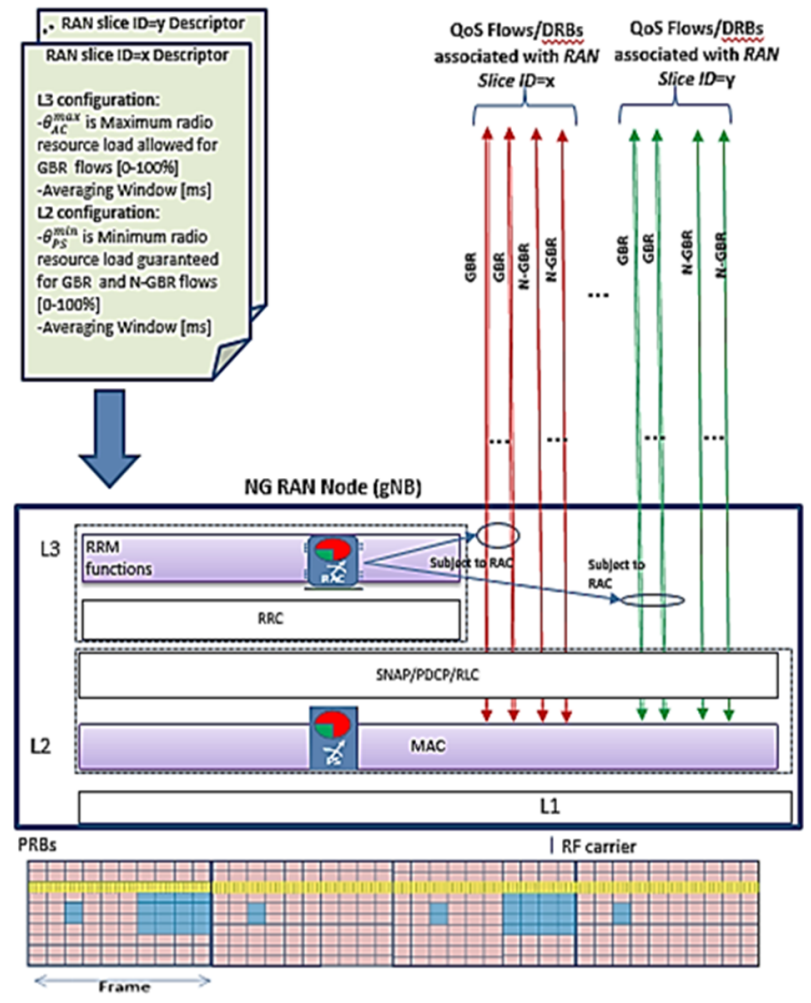

Fig. 1. RAN slicing configuration model

Focusing now on L2, this layer comprises a Medium Access Control (MAC) sub-layer for multiplexing and scheduling the 
packet transmissions of the DRBs over a set of transport channels exposed by L1. Moreover, L2 embeds a number of processing functions configurable on a per-DRB basis for e.g. segmentation, Automatic Repeat request (ARQ) retransmissions, compression and ciphering (i.e. Radio Link Control [RLC] and Packet Data Convergence Protocol [PDCP]). In the NR specifications, an additional L2 sub-layer named Service Data Adaptation Protocol (SDAP) is included to map the DRBs and the traffic flows managed by the $5 \mathrm{GC}$, referred to as QoS Flows[14]. Therefore, considering that the current MAC operation is based on individual UE and DRBspecific QoS profiles, it is necessary to define the Packet Scheduling (PS) behaviors to be enforced on the traffic aggregate of DRBs associated with a particular RAN slice and to specify the capability set of the applicable L2 sub-layers processing functions. Accordingly, this paper considers as the main means of RAN slices' control related to L2 a parameter that specifies the minimum \% of PRBs that the PS guarantees to the slice for allocating transmissions of both GBR and nonGBR bearers. The limit is expressed as a minimum \% because the actual value of PRBs used by a RAN slice can exceed this value at specific points of time provided that other RAN slices are not consuming all their PRBs. This parameter is denoted as $\theta_{P S}^{\min }(s)$ for the slice $s$.

In the following, we explain how the $\theta_{A C}^{\max }(s)$ and $\theta_{P S}^{\min }(s)$ are accounted within the slice-aware RAC and PS functions.

\section{A. Slice-aware Radio Admission Control (RAC)}

RAC decisions have to take into account the number of PRBs committed to the RAN slices. At the same time, RAC should consider the actual PRBs utilization according to the ARP parameter associated with each QoS flow. ARP defines the relative importance of admitting, keeping or terminating a QoS flow. 3GPP specifications define priority levels from 1 to 15 for the ARP, being 1 the highest level of priority. Therefore, when a new GBR bearer request is received in the NG-RAN for an $s$-th slice, the following condition is checked:

$\theta_{o c c}\left(A R P_{i}, s\right)+\theta_{\text {new }}(i, s) \leq \theta_{A C}^{\max }(s)$

Where $\operatorname{\theta occ}(A R P i, s)$ measures the average number of PRBs (with the representatives of PRBs already using in slice) used by the GBR bearers of slice $s$ that have an ARP value equal or lower than $A R P i, \theta$ new $(i, s)$ is the estimated PRBs needed of the new bearers, and $\theta_{A C}^{\max }(s)$ is used as the admission control threshold in the $s$-th slice, and $\theta_{n e w}(i, s)$ could be calculated based on the formula:

$\theta_{\text {new }}(i, s)=\frac{R_{i, s}}{r \cdot S_{i, s}}$

Where $R i, s$ is the required bit rate in $i$-th service of the $s$-th slice, $r$ is the bandwidth of one PRB, and $S i, s$ is the spectrum efficiency in $i$-th service of the $s$-th slice.

\section{B. Slice aware Packet Scheduling (PS)}

For the scheduling part, the resource distribution at L2 shall first guarantee the admitted GBR bearers get the necessary PRBs to meet its rate requirements. Then, the PS can allocate the remaining PRBs not consumed by GBR traffic to serve the NonGBR bearers according to their $5 \mathrm{QI}$ value. $5 \mathrm{QI}$ is a scalar that defines the specific QoS characteristics in terms of, among others, a priority level to be enforced by the PS. Therefore, in order to establish how PRBs are distributed by the PS, let us assume that after assigning the PRBs to GBR bearers, there are $\mathrm{N}$ available PRBs that have to be distributed among $k$ Non-GBR bearers, and $\varphi i$ is the priority level of the $i$-th bearer based on its 5QI in slice $s$. Then, the average number of PRBs that will be assigned to the $i$-th radio bearer and the formula for calculating the PRBs assign to $i$-th Non-GBR service in slice $s$ is:

$$
\mathrm{N}_{i, s}=\left(\theta_{P S}^{\min }(s)-N_{a s s, i, s}\right) \cdot \frac{1 / \varphi_{i}}{\sum_{j=1}^{\kappa} 1 / \varphi_{j}}
$$

For the separately slicing part, and $k$ represents the NonGBR bearers of a specific slice $s, \mathrm{~N}_{i, s}$ is the available PRBs for Non-GBR service $i$ in slice $s . \theta_{P S}^{\min }(s)$ is minimum PRBs that the PS guarantees for the slice $s$ and $\mathrm{N} a s s, i, s$ is the sum assigned PRBs for the $i$-th GBR services in slice $s$. At the same time, if one slice has no active Non-GBR bearers, the remaining PRBs of it may be allocated the other slices, which possessing NonGBRs. And this process will accord the priority level which is presented as 5QI value of different services.

\section{EVAluation ScENARIO}

Let's consider an operational scenario where a NG-RAN is used to provide both $\mathrm{CO}$ services to the general public and $\mathrm{MC}$ services for PS organizations. The Commercial services include two different user services, the Premium and Basic service. Each of them includes a GBR video service and a Non-GBR data service. The Premium Video service will supply for high data quality while the Basic video service provides common data quality. The MC services include two GBR services and one Non-GBR service; they are named MC Video, MC PTT, and MC Data service respectively. The characteristics of these services in terms of QoS parameters (i.e. bearer type,5QI $(\varphi)$, GFBR) as well as traffic mix composition are given in Table 1.

TABLE 1. SERVICES OF EACH RAN SLICE

\begin{tabular}{|c|c|c|c|c|c|c|}
\hline $\begin{array}{c}\text { Service } \\
\text { Type }\end{array}$ & Service & Type & $\begin{array}{c}\text { 5QI } \\
(\varphi)\end{array}$ & ARP & GFBR & $\begin{array}{c}\text { Traffic } \\
\text { demand } \\
\text { mix }\end{array}$ \\
\hline \multirow{2}{*}{ CO } & $\begin{array}{c}\text { Premium } \\
- \text { Video } \\
\text { HD }\end{array}$ & GBR & $2(40)$ & 2 & $\begin{array}{c}10 \\
\mathrm{Mb} / \mathrm{s}\end{array}$ & $10 \%$ \\
\cline { 2 - 7 } & $\begin{array}{c}\text { Premium } \\
- \text { Data }\end{array}$ & $\begin{array}{c}\text { Non- } \\
\text { GBR }\end{array}$ & $6(60)$ & 2 & $\mathrm{~N} / \mathrm{A}$ & $20 \%$ \\
\cline { 2 - 7 } & $\begin{array}{c}\text { Basic- } \\
\text { Video }\end{array}$ & GBR & $2(40)$ & 3 & $1 \mathrm{Mb} / \mathrm{s}$ & $25 \%$ \\
\cline { 2 - 7 } & $\begin{array}{c}\text { Basic - } \\
\text { Data }\end{array}$ & $\begin{array}{c}\text { Non- } \\
\text { GBR }\end{array}$ & $8(80)$ & 3 & $\mathrm{~N} / \mathrm{A}$ & $45 \%$ \\
\hline \multirow{2}{*}{ MC } & $\begin{array}{c}\text { MC } \\
\text { Video }\end{array}$ & GBR & $2(40)$ & 2 & $5 \mathrm{Mb} / \mathrm{s}$ & $20 \%$ \\
\cline { 2 - 7 } & MC PTT & GBR & $65(7)$ & 1 & $10 \mathrm{~kb} / \mathrm{s}$ & $50 \%$ \\
\hline
\end{tabular}




\begin{tabular}{|l|c|c|c|c|c|c|}
\hline & MC Data & $\begin{array}{c}\text { Non- } \\
\text { GBR }\end{array}$ & $\begin{array}{c}70(55 \\
)\end{array}$ & 3 & N/A & $30 \%$ \\
\hline
\end{tabular}

Let's consider a NG-RAN deployment according to the parameterization presented in Table 2 . The deployment assumes a gNB with a single cell configured with a bandwidth of 100 $\mathrm{MHz}$, and average cell capacity is $336 \mathrm{Mb} / \mathrm{s}$, which computed as the product of channel bandwidth and the average spectral efficiency (obtained from the simulation that is $3.36 \mathrm{~b} / \mathrm{s} / \mathrm{Hz}$ ). Finally, we only consider the downlink direction.

TABLE 2. CELL DEPLOYMENT AND TRAFFIC MODELLING

\begin{tabular}{|c|c|}
\hline Parameter & Value \\
\hline Cell radius & $115 \mathrm{~m}$ \\
\hline Path loss and shadowing model & $\begin{array}{l}\text { Urban micro-cell model with } \\
\text { hexagonal layout } \\
\text { in[15]) }\end{array}$ \\
\hline Shadowing standard deviation & $\begin{array}{l}3 \mathrm{~dB} \text { in Line Of Sight (LOS) and } 4 \\
\mathrm{~dB} \text { in Non Line Of Sight (NLOS) } \\
{[15]}\end{array}$ \\
\hline Base station antenna gain & $5 \mathrm{~dB}$ \\
\hline Frequency & $3.6 \mathrm{GHz}$ \\
\hline Transmitted power per PRB & $16.6 \mathrm{dBm}$ \\
\hline Number of PRBs & 275 \\
\hline UE noise figure & $9 \mathrm{~dB}$ \\
\hline $\begin{array}{l}\text { Link-level model to map Signal to } \\
\text { Interference and Noise Ratio and bit } \\
\text { rate }\end{array}$ & $\begin{array}{l}\text { Model in section A.1 of [16] with } \\
\text { maximum spectral efficiency } 8.8 \\
\text { b/s/Hz. }\end{array}$ \\
\hline $\begin{array}{l}\text { Planned average session generation } \\
\text { rate }(\hat{\lambda})\end{array}$ & $\begin{array}{l}\lambda_{C O}=1 \text { sessions } / \mathrm{s} \\
\lambda_{M C}=0.5 \text { sessions } / \mathrm{s}\end{array}$ \\
\hline Average session duration (T) & $\mathrm{T}=\mathrm{T} C O=\mathrm{T}_{M C}=120 \mathrm{~s}$ \\
\hline $\begin{array}{l}\text { Activity factor of } \\
\text { sessions }(\delta)\end{array}$ & $\delta=\delta c o=\delta M c=0.2$ \\
\hline
\end{tabular}

Let us consider that the planned load per cell is computed based on the traffic demand parameters provided in Table1 and 2. Accordingly, the planned load for all GBR services, including both $\mathrm{CO}$ and $\mathrm{MC}$, is $210 \mathrm{Mb} / \mathrm{s}$. It is almost $62 \%$ of the average cell capacity. Based on traffic mixes mentioned in Table1, the cell supports a traffic load $(\lambda)$, which is generated in the simulation scenario and computed based on the plan average session generation $(\hat{\lambda})$ factored by an overload index $(\rho=\lambda / \hat{\lambda})$. Each session corresponds to one QoS flow for one DRB associated with a UE at a random position following a uniform distribution within the cell radius. In this paper, the RAN will be sliced into two slices, the one is slice1, which is mainly supply $\mathrm{CO}$ services, and the other slice 2 is for MC services. Based on the planned load, two different RAN slicing configurations will be studied. The first configuration shares a slice for both $\mathrm{CO}$ and $\mathrm{MC}$ services, and the second configuration separates slices for $\mathrm{CO}$ and $\mathrm{MC}$ services. Planned load for CO GBR services is $150 \mathrm{Mb} / \mathrm{s}$, at the same time, planned load for MC GBR services is $60 \mathrm{Mb} / \mathrm{s}$. All the details and specific description of these two configurations is illustrated in Table 3:

TABLE 3. SLICING CONFIGURATION

\begin{tabular}{|l|l|l|}
\hline $\begin{array}{l}\text { Slicing } \\
\text { configuration } \\
\text { parameters }\end{array}$ & $\begin{array}{l}\text { Slicing } \\
\text { Configuration \#1 }\end{array}$ & Slicing Configuration \#2 \\
\hline
\end{tabular}

\begin{tabular}{|l|c|c|c|}
\hline Slice Identifier & RAN Slice ID=0 & $\begin{array}{l}\text { RAN Slice } \\
\text { ID=1 for } \\
\text { CO }\end{array}$ & $\begin{array}{l}\text { RAN Slice } \\
\text { ID=2 for MC }\end{array}$ \\
\hline $\begin{array}{l}\text { L3 configuration } \\
\left(\theta_{A C}^{\max }(s)\right)\end{array}$ & $70 \%$ & $45 \%$ & $25 \%$ \\
\hline $\left.\begin{array}{l}\text { L2 configuration } \\
\left(\theta_{P S}^{\min }(s)\right.\end{array}\right)$ & N/A & $65 \%$ & $35 \%$ \\
\hline
\end{tabular}

\section{Performance Evaluation}

We are using Matlab as simulation tools and the results are provided in this section for two configurations. For ARP configuration, different ARP values are set in MC video services in order to compare the impact of different priorities on resource allocation.

\section{GBR services}

The main Key Performance Indicator (KPI) used to assess the performance for GBR services is the blocking rate, which is calculated as the percentage of GBR DRB requests rejected by the RAC. Fig. 2 shows the blocking rate observed for Premium Video HD, Basic Video, and MC Video services. The blocking rate values have been obtained from the execution of 5 simulations, each lasting for 20000 seconds (the standard deviation of the computed values is observed to be below $5 \%$ ). The overload index is set in the range of $0.5 \leq \rho_{C O}, \rho_{M C} \leq 2$. The ARP values used for all the services are those provided in Table 1, except for the MC Video service for which two different settings are considered: 2 (default, as set in Table 1) and 1 (the highest priority). To facilitate the reading of Fig.2, blocking rate values are colored in red for rates above $10 \%$, orange for rates between $5 \%$ and $10 \%$, yellow for values between $2 \%$ to $5 \%$ and green when the blocking rate is below $2 \%$.

Fig. 2 shows the results for the two slicing configurations and the different ARP values considered for the MC Video service. As expected, the blocking rate is kept low for overload indexes below the planned ones $\left(\rho_{C O}<1\right.$ and $\left.\rho_{M C}<1\right)$ but exhibits a growing trend when there is an overload situation in any of the group of services, $\left(\rho_{C O}>1\right.$ or $\left.\rho_{M C}>1\right)$. We can find blocking rate is kept low for $\mathrm{CO} / \mathrm{MC}$ services when overload index below 1, and it exhibits an increasing trend with overload index growing up. Let us consider slicing Configuration\#1 with $\mathrm{MC}$ Video ARP=2 as the benchmark. For instance, Premium and $\mathrm{MC}$ video service have a similar change in blocking rate when overload index of both services are less than 1. That because $\mathrm{MC}$ and Premium Video are treated equally by RAC without considering the load unbalances. But when these two overload indexes are all exceeding 1 , that means the PRBs for the radio access bearers will be saturation, so the blocking rate is increasing sharply. And the blocking rate of the Basic video is the highest in these three services, that because it has the lowest priority.

Comparing now the case of slicing Configuration\#1 with $\mathrm{MC}$ Video $\mathrm{ARP}=1$ with benchmark solution, a blocking rate of Premium video in the former configuration is higher than the later, that because $\mathrm{MC}$ video has a higher priority than Premium Video, so congestion and rejection probability of Premium Video service in ARP Configuration\#2 is higher than ARP 


\begin{tabular}{|c|c|c|c|c|c|c|c|c|c|c|c|c|c|c|c|c|c|}
\hline \multicolumn{18}{|c|}{ Slicing Configuration\#1, ARP values from Table 1} \\
\hline & \multicolumn{5}{|c|}{ Premium Video HD(ARP=2) } & & \multicolumn{5}{|c|}{ Basic Video(ARP=3) } & & \multicolumn{5}{|c|}{ MC Video (ARP=2) } \\
\hline 1.5 & 0 & 0.5 & 4.5 & 14.1 & 16.6 & 1.5 & 0 & 11.2 & 13.1 & 28.8 & 46.9 & 1.5 & 0 & 0 & 1.3 & 9.6 & 13.3 \\
\hline 1.25 & 0 & 0 & 3.1 & 7.9 & 12.8 & 1.25 & 0 & 0 & 7.5 & 21.5 & 34.8 & 1.25 & 0 & 0 & 0.3 & 3.6 & 7.6 \\
\hline 1 & 0 & 0 & 0 & 4.5 & 10.6 & 1 & 0 & 0 & 1.4 & 16.2 & 26.4 & 1 & 0 & 0 & 0 & 1.4 & 5.4 \\
\hline 0.75 & 0 & 0 & 0 & 4.4 & 8.2 & 0.75 & 0 & 0 & 0 & 8.3 & 23.9 & 0.75 & 0 & 0 & 0 & 0.3 & 4.8 \\
\hline \multirow[t]{2}{*}{0.5} & 0 & 0 & 0 & 2.8 & 5.8 & 0.5 & 0 & 0 & 0 & 6.6 & 20.8 & 0.5 & 0 & 0 & 0 & 0 & 1.8 \\
\hline & 0.5 & 0.75 & 1 & 1.25 & 1.5 & & 0.5 & 0.75 & 1 & 1.25 & 1.5 & & 0.5 & 0.75 & 1 & 1.25 & 1.5 \\
\hline \multicolumn{18}{|c|}{ Slicing Configuration\#1. ARP values from Table 1, except ARP=1 for MC Video. } \\
\hline & \multicolumn{5}{|c|}{ Premium Video HD(ARP=2) } & & \multicolumn{5}{|c|}{ Basic Video(ARP=3) } & & \multicolumn{5}{|c|}{ MC Video (ARP=1) } \\
\hline 1.5 & 0 & 0.6 & 6.9 & 10.9 & 21.9 & 1.5 & 0 & 11.2 & 13.5 & 20.9 & 42 & 1.5 & 0 & 0 & 0 & 0 & 0 \\
\hline 1.25 & 0 & 0 & 5.4 & 8.7 & 16.7 & 1.25 & 0 & 0 & 13.5 & 20.1 & 39.4 & 1.25 & 0 & 0 & 0 & 0 & 0 \\
\hline 1 & 0 & 0 & 0 & 3.1 & 15.1 & 1 & 0 & 0 & 1.4 & 17.9 & 33.8 & 1 & 0 & 0 & 0 & 0 & 0 \\
\hline 0.75 & 0 & 0 & 0 & 2.9 & 10.4 & 0.75 & 0 & 0 & 0 & 9.5 & 26.2 & 0.75 & 0 & 0 & 0 & 0 & 0 \\
\hline \multirow[t]{2}{*}{0.5} & 0 & 0 & 0 & 2.8 & 7.4 & 0.5 & 0 & 0 & 0 & 8.3 & 24.5 & 0.5 & 0 & 0 & 0 & 0 & 0 \\
\hline & 0.5 & 0.75 & 1 & 1.25 & 1.5 & & 0.5 & 0.75 & 1 & 1.25 & 1.5 & & 0.5 & 0.75 & 1 & 1.25 & 1.5 \\
\hline \multicolumn{18}{|c|}{ Slicing Configuration\#2. ARP values from Table 1 , except $A R P=1$ for $M C$ Video } \\
\hline & \multicolumn{5}{|c|}{ Premium Video HD(ARP=2) } & & \multicolumn{5}{|c|}{ Basic Video(ARP=3) } & & \multicolumn{5}{|c|}{ MC Video (ARP=1) } \\
\hline 1.5 & 0 & 0.6 & 1.8 & 8.2 & 13.2 & 1.5 & 0 & 0 & 1.8 & 17.2 & 31.9 & 1.5 & 4.8 & 8.4 & 9.1 & 9.3 & 12.5 \\
\hline 1.25 & 0 & 0 & 0.5 & 4.3 & 9.1 & 1.25 & 0 & 0 & 0.4 & 8.7 & 21.7 & 1.25 & 2.9 & 3.1 & 4.3 & 4.6 & 4.6 \\
\hline 1 & 0 & 0 & 0.4 & 3.9 & 8.3 & 1 & 0 & 0 & 0 & 4.6 & 14.9 & 1 & 0 & 0 & 0 & 0 & 0 \\
\hline 0.75 & 0 & 0 & 0 & 3.5 & 4.5 & 0.75 & 0 & 0 & 0 & 4.4 & 11.4 & 0.75 & 0 & 0 & 0 & 0 & 0 \\
\hline \multirow[t]{2}{*}{0.5} & 0 & 0 & 0 & 2.3 & 3.3 & 0.5 & 0 & 0 & 0 & 2.4 & 11.2 & 0.5 & 0 & 0 & 0 & 0 & 0 \\
\hline & 0.5 & 0.75 & 1 & 1.25 & 1.5 & & 0.5 & 0.75 & 1 & 1.25 & 1.5 & & 0.5 & 0.75 & 1 & 1.25 & 1.5 \\
\hline
\end{tabular}

Fig.2. Blocking rate of (a) Premium Video HD service, (b) Basic Video service, (c) MC Video service with different configurations

Configuration\#1. At the same time, with the MC blocking rate decreased, the Premium and Basic video service's blocking rate is increasing slightly, that because more PRBs allocated to MC video service, and a little PRBs left for Premium and Basic video services.

Due to Slicing Configuration\#2 with $\mathrm{MC}$ Video $\mathrm{ARP}=1$ have similar performance for all services, therefore, we just analyze Slicing Configuration\#2, ARP Configuration\#2. Focus on Premium and Basic video service, we can find when the overload index of MC is increasing from 0.5 to 1.5 and the overload index of $\mathrm{CO}$ is below 1 , the blocking rate is low, so the color is green, but for $\mathrm{MC}$ video service is opposite, the reason is Configuration of Slicing will make a distinguishing in RAN slice at L3 and just configure a whole admission control of $45 \%$ PRBs for GBRs DRBs in CO slice, $25 \%$ PRBs for GBR DRBs will be allocated to MC slice. Therefore, the overload of Premium and Basic video service will not impact on the MC video service, vice versa. It illustrates the slices are isolated successfully. Comparing Slicing Configuration\#2, ARP Configuration\#2 with Slicing Configuration\#1, ARP Configuration\#2 and benchmark solution, we can find the former blocking rate of $\mathrm{MC}$ video service under overload situation of is higher than the later, even though the ARP value is the same. The reason is that the behavior of prioritization mechanisms has a little influence on the performance under the isolation of RAN slicing.

\section{Non-GBR services}

Non-GBR services just make use of PRBs which are available after having performed the PRBs allocation to the GBR services. Accordingly, the KPI assessed for Non-GBR services is the average throughput obtained. Fig. 3 shows the average throughput of Premium and Basic data service in RAN
Slice1 and MC data service in RAN Slice2. Simulation work is underlying a situation of MC video ARP value is equal to 2 . The average throughput obtained from the execution of 5 simulations, each lasting for 20000 seconds (the standard deviation of the computed values is less than $4.5 \mathrm{Mb} / \mathrm{s}$ ). We set Slicing Configuration\#1 as the benchmark solution, as expected, throughput is kept high for overload index below 1 but exhibits a decreasing when there is an increasing trend in any of the group of services. There are two main reasons, the one is the load increase brings the increasing of the number of GBR sessions of RAN Slice1, and it will lead to leaving fewer PRBs available to the Non-GBR service, the other is Non-GBR data session will increase when the loads growing up, therefore, the available PRBs will be distributed among lots of radio bearers.

It is observed in Fig. 3 that, when the overload index in both tenants ( $\mathrm{CO}$ and $\mathrm{MC}$ ) are much higher than 1, the throughput is close to $5 \mathrm{Mb} / \mathrm{s}$ in these three services. Let's focus on the service of Fig.(a), (b)and (c), with the overload index is decreased, there is an increasing trend of the throughput. For instance, when the overload index of $\mathrm{CO}$ and $\mathrm{MC}$ are all 0.5 , the obtained throughput is about $24 \mathrm{Mb} / \mathrm{s}, 20 \mathrm{Mb} / \mathrm{s}$ and $25 \mathrm{Mb} / \mathrm{s}$ respectively, which can be seen that the highest value could be got from low overload index of both tenants. However, the Fig.(c) get the highest throughput compared to Fig.(a) and (b), that because of the 5QI value of Fig.(c) is lowest (highest priority) in those three services. From Fig.(d), we can find the trend of throughput is decreased sharply and the distance of the curves are closer when compared to Fig(a), the reason is that the use of slicing technique could get a good isolation for tenant, and the changing of overload index in $\mathrm{MC}$ will not affect the $\mathrm{CO}$ services' performance, this situation is the same applying to Fig.(b) and (e). For Fig.(f), the throughput of MC data service 
is changing smoothly under MC overload index is exceeding 1 when compared to Fig.(c), that because slicing techniques will guarantee at least $35 \%$ PRBs allocated to MC service, the overload of $\mathrm{CO}$ data services will not impact on the performance of $\mathrm{MC}$ data service.

\section{CONCLUSION}

RAN slicing is demonstrated to be a suitable method to guarantee the necessary isolation when multiplexing $\mathrm{MC}$ and $\mathrm{CO}$ services over the same network. While the proper setting of ARP/5QI parameters used for controlling QoS is a valid
[4] 3GPP TS 22.281 V16.0.0, "Technical Specification Group Services and System Aspects, Mission Critical Video services", (2018-09)

[5] 3GPP TS 22.282 V16.4.0," Technical Specification Group Services and System Aspects, Mission Critical Data services" (2018-12)

[6] Haya Shajaiah, Ahmed Abdel-Hadi, Charles Clancy," Spectrum Sharing between Public Safety and Commercial Users in 4G-LTE", 2014 International Conference on Computing, Networking and Communications (ICNC)

[7] Suresh Borkar, Dennis Roberson, Ken Zdunek," Priority Access for public safety on shared commercial LTE networks" 2011 Technical Symposium at ITU Telecom World (ITU)

[8] Chafika Tata, Michel Kadoch," Courteous Priority Access to the Shared Commercial Radio for Public Safety in LTE Heterogeneous Networks",

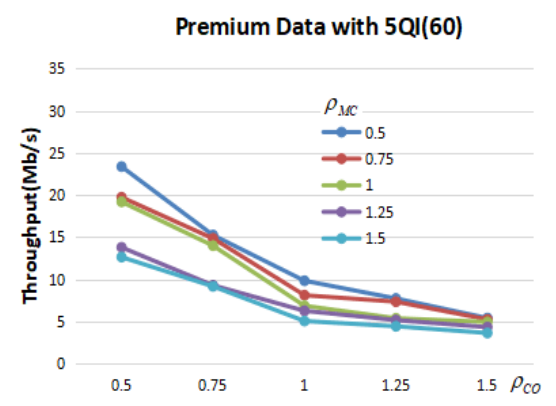

(a)

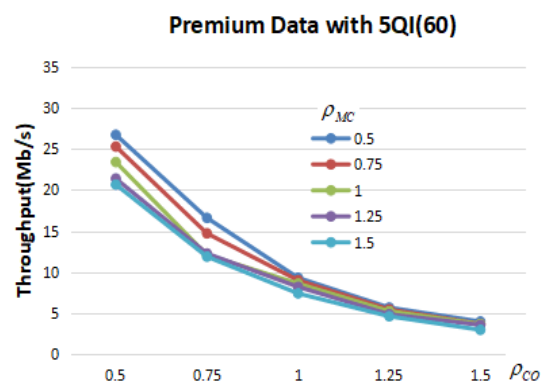

(d)
Slicing Configuration\#1

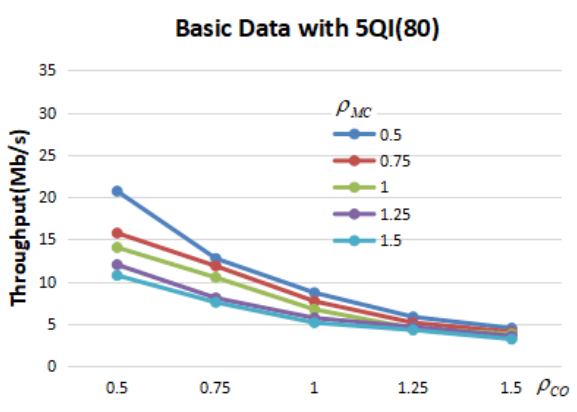

(b)
Slicing Configuration\#2

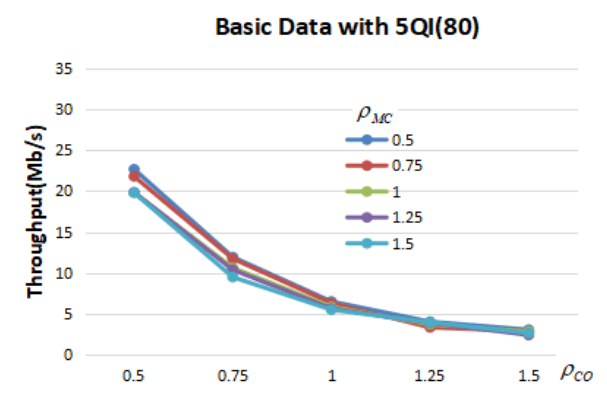

(e)

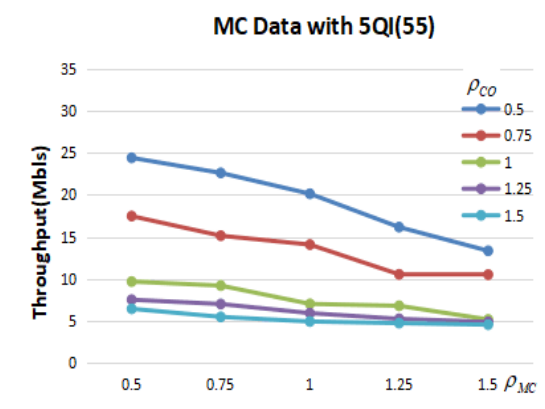

(c)

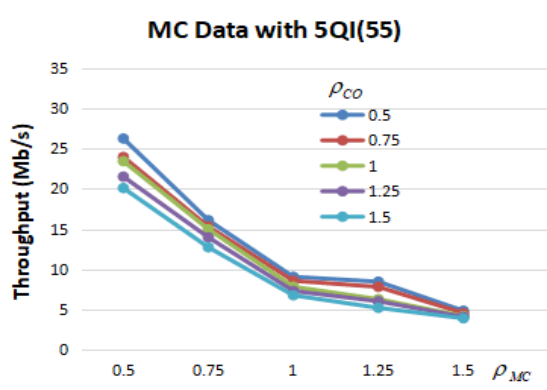

(f) framework to provide protection to $\mathrm{MC}$ service configured with preferential $\mathrm{ARP} / 5 \mathrm{QI}$ settings, $\mathrm{CO}$ traffic rest unprotected in front of a surge of MC traffic when no slicing is used. By relying on slice-aware admission control and packet scheduling functions parameterized with the desired radio load guarantees for $\mathrm{MC}$ and $\mathrm{CO}$ traffic, the solution analyzed in this paper provides isolation for the GBR and Non-GBR services.

\section{ACKNOWLEDGEMENT}

This work has been supported by the Spanish Research Council, FEDER funds under SONAR 5G grant (ref. TEC201782651-R) and the program of China Scholarships Council (No. 201808390034).

\section{REFERENCES}

[1] NGMN Alliance,"5G White Paper", February 2015

[2] Ramon Ferrus, Oriol Sallent, Gianmarco Baldini, Leonardo Goratti," LTE: the technology driver for future public safety communications", IEEE Communications Magazine, October 2013. DOI: 10.1109/MCOM.2013.6619579

[3] 3GPP TS 22.179 V16.4.0, "Technical Specification Group Services and System Aspects; Mission Critical Push To Talk (MCPTT)” (2018-12)
2014 International Conference on Future Internet of Things and Cloud. DOI: 10.1109/FiCloud.2014.46

[9] Hossam Farag, Mikael Gidlund, Patrik Oesterberg "PR-CCA MAC: A Prioritized Random CCA MAC Protocol for Mission-Critical IoT Applications" 2018 IEEE International Conference on Communications (ICC), DOI: 10.1109/ICC.2018.8423018

[10] Dania Marabiss, Romano Fantacci," Heterogeneous Public Safety Network Architecture Based on RAN Slicing", IEEE Access, DOI:10.1109/ACCESS.2017.2768800

[11] Marko Hoyhtya, Kalle Lahetkangas, Jani Suomalainen, Mika Hoppari, Kaisa Kujanpaa, Kien Trung Ngo, Tero Kippola, Marjo Heikkila, Harri Posti, Jari Maki, Tapio Savunen, Ari Hulkkonen, Heikki Kokkinen," Critical Communications Over Mobile Operators' Networks: 5G Use Cases Enabled by Licensed Spectrum Sharing, Network Slicing and QoS Control", IEEE Access. DOI:10.1109/ACCESS.2018.2883787

[12] J. Perez-Romero, O. Sallent, R. Ferrus, R. Agusti," On the configuration of Radio resource management in a sliced RAN". NOMS 2018,10.1109/NOMS.2018.8406280

[13] R. Ferrus, O. Sallent, J. Perez-Romero, R. Agusti," On 5G Radio Access Network Slicing: Radio Interface Protocol Features and Configuration", Communication Magazine, September 2017.

[14] 3GPP TS 38.300 V0.4.1, "NR; NR and NG-RAN Overall Description; Stage 2 (Release 15)", June 2017. 
[15] 3GPP TR 36.814 v9.0.0, "E-UTRA: Further advancements for E-UTRA physical layer aspects (Release9)" March 2010
[16] 3GPP TR 36.942 v12.0.0, "Radio Frequency (RF) system scenarios", September, 2014. 